\title{
DIVERSIDAD FLORISTICA DE LOS PETENES DE CAMPECHE
}

\author{
Rafael Duran Garcia \\ Centro de Investigación Científica de Yucatán \\ Apartado Postal 87, Cordemex, Mérida \\ 97310, Yucatán, México
}

\section{RESUMEN}

En el presente trabajo se discute brevemente el uso de distintos índices y modelos teóricos en el estudio de la riqueza florística, la diversidad y la distribución de abundancias de las especies en las comunidades vegetales.

Se ensaya la aplicación de estas herramientas descriptivas en cuatro comunidades insulares de vegetación selvática (petenes) del noroeste del estado de Campeche.

Se plantea el uso de los números propuestos por Hill como mejores indicadores de la diversidad.

Los resultados indican que la dominancia disminuye y la diversidad se incrementa conforme aumenta el tamaño de los petenes.

En cuanto a la distribución de abundancias, los petenes parecen ajustarse al modelo de Motomura, lo que apoyaría la idea de que son comunidades que se encuentran en distintos estadios de un proceso sucesional.

\section{ABSTRACT}

The use of several indices and theoretical models for the study of floristic diversity, species abundance and species richness is discussed.

These descriptive methods are applied to four patches of tropical vegetation known as hammocks or "petenes" in NW Campeche state, Mexico.

It is suggested that the Hill index best indicates vegetation diversity.

The results show that dominance decreases as island size and diversity increase.

The distributions of species abundance conform to Motomura's model, suggesting that these communities represent different successional stages.

\section{INTRODUCCION}

Los estudios ecológicos a nivel de las comunidades naturales se han centrado, durante mucho tiempo, en el aspecto descriptivo de las mismas, empleando un conjunto de criterios que van desde las caracterizaciones fisonómicas cualitativas hasta el uso de diversos índices de riqueza, diversidad, distribución espacial, etc.

La forma más simple de caracterizar cuantitativamente a una comunidad es mediante el conteo del número de especies presentes. Esta medida se conoce como "Riqueza de Especies" y en ocasiones ha sido utilizada como un indicador de la diversidad. 
Por medio de otros métodos, se ha intentado evaluar la importancia de cada una de las especies que se presentan en la comunidad. Siguiendo distintos criterios, se han empleado parámetros como son la biomasa, la cobertura, el área basal, la densidad y la constancia con que se presenta cada especie. Según Whittaker (1972), cualquiera de éstos puede ser usado como valor de importancia, dependiendo de qué parámetro considere el autor como el más importante. Algunos investigadores proponen el empleo de índices que combinan varios de estos parámetros, como es el Valor de Importancia Relativa (VIR) de Curtis (1959), el cual consiste en la sumatoria de los valores relativos de densidad, frecuencia y dominancia.

Otros métodos intentan evaluar la heterogeneidad de las comunidades a través de un concepto dual, que involucra tanto la riqueza de especies como la abundancia de cada una de éstas. Así se ha generado un conjunto de "Indices de Diversidad o Heterogeneidad" (Shannon-Wiener, Simpson, Brillouin, etc.), que son sensibles en distintos grados a uno u otro de los parámetros (Peet, 1974; Hill, 1973). Al aplicar estos índices se obtienen como resultado cifras logarítmicas difíciles de interpretar, por lo que Hill (1973) propone algunas modificaciones con el fin de brindar una información más clara en términos de "especies aparentes" en la comunidad, eliminando así las expresiones logarítmicas.

Un método gráfico que consiste en ordenar a las especies en rangos de mayor a menor, considerando la abundancia de cada una, nos muestra una imagen de la curva que une las abundancias de las mismas. Estas gráficas nos brindan información acerca del nivel de dominancia que se presenta en la comunidad y se conocen como "Curvas de Distribución de Abundancias" (Pielou, 1975). Algunos otros autores las han denominado "Curvas de Dominancia-Diversidad" (Whittaker, 1972; Bazzaz, 1975; Peet, 1974). Las gráficas pueden ser elaboradas considerando como indicador de la abundancia $\left(\mathrm{p}_{\mathrm{i}}\right)$ cualquier parámetro como cobertura, biomasa y densidad, incluso el Valor de Importancia (VIR).

El presente trabajo tiene como objetivo central evaluar y discutir brevemente los métodos mencionados, tomando como ejemplo cuatro comunidades insulares (petenes) localizadas en el estado de Campeche, México. Al mismo tiempo se pretende mediante este ensayo, hacer una breve aproximación del análisis del comportamiento de las islas de vegetación, con relación al número de especies que pueden sostener, su diversidad (medida a través de varios índices) y a sus curvas de dominancia. Por último se pretende ajustar un modelo teórico que nos permita plantear algunas hipótesis.

\section{COMUNIDADES DE ESTUDIO}

Los petenes son pequeñas "islas" de vegetación arbórea, principalmente de selva mediana perennifolia y subperennifolia y de manglar, que se encuentran inmersas en medio de amplias zonas inundables de tipo pantanoso, conocidas como "marismas" (Barrera, 1982; Rico-Gray, 1982; Olmsted et al., 1983). Estas marismas están constituídas en esencia de pastizales y manglares enanos fuertemente influenciados por los movimientos de las aguas del mar. Los petenes se distribuyen en todo el borde de la Península de Yucatán, pero son particularmente abundantes en la porción centro oriental de Quintana Roo y en el noroeste del estado de Campeche. 
Por su condición insular, su tamaño pequeño y por las condiciones limitantes que imperan en ellas, las comunidades vegetales presentes en los petenes son relativamente sencillas, en cuanto al número de especies y a su estructura (Durán, 1987). Estas características nos brindan la oportunidad de ensayar allí, el comportamiento de distintos índices empleados en la descripción de las comunidades y explorar acerca de la sensibilidad de cada uno de éstos, a los cambios en el número de especies vegetales que se presentan y en la abundancia de las mismas.

\section{METODOLOGIA}

Este trabajo se centra en el análisis de los petenes del estado de Campeche. Durán (1987) describe con detalle la vegetación de estas islas y son los datos relativos a los árboles de dicho trabajo los que serán retomados para el presente ensayo. Los petenes aquí analizados corresponden a los números 3, 4, 5 y 6 de Durán (1987).

En cada uno de los petenes seleccionados se estableció un cuadro de muestreo de $800 \mathrm{~m}^{2}$ (40 x $20 \mathrm{~m}$ ), que se subdividió en 8 subcuadros de $100 \mathrm{~m}^{2}$ cada uno. Se censó a todos los individuos enraizados dentro de los límites marcados.

La categorización de los organismos muestreados se hizo en forma artificial, considerando como árbol a todo individuo mayor o igual a $3 \mathrm{~m}$ de altura y como arbusto a aquellos entre $60 \mathrm{~cm}$ y $2.99 \mathrm{~m}$. Las plántulas y hierbas menores de $60 \mathrm{~cm}$ no fueron consideradas.

Con la información recopilada se determinaron los valores de importancia mediante el método de Curtis (1959). Para cada petén se calculó la riqueza de especies arbóreas, la diversidad florística (según los índices de Shannon-Wiener y de Simpson) y el valor de equidad. Se realizó la transformación de estos índices, obteniendo los llamados números de Hill. Se generó una curva de abundancia-diversidad para todas las comunidades y a cada una de éstas se le ajustaron, además, los modelos teóricos de Motomura y de MacArthur (ver Pielou, 1975).

\section{RESULTADOS}

El Cuadro 1 muestra algunas de las características de los petenes seleccionados en el presente estudio y los valores de diversidad obtenidos mediante el uso de los distintos índices para la comunidad arbórea de cada uno. Cabe señalar que el tamaño de los petenes aumenta del número 1 al 4 .

En cuanto a la riqueza florística y considerando que se trata de asociaciones vegetales de tipo selvático, se observa que todas las comunidades presentan un reducido número de especies arbóreas y que el petén 2 resultó ser el más pobre.

Por otra parte, se muestra que los "números de especies aparentes" obtenidos mediante el método de Hill (1973), a partir de los índices de Shannon-Wiener $\left(\mathrm{N}_{1}\right)$ y de Simpson $\left(\mathrm{N}_{2}\right)$, se comportan de manera distinta a la riqueza florística. Con los primeros, se obtiene una menor diversidad para el petén 1, ya que ambos índices consideran tanto el número de especies presentes, como la distribución de abundancia de las mismas, la cual ha sido estimada a través del valor de equidad $(E)$. La figura 1 muestra en forma gráfica 
Cuadro 1. Algunas características de los petenes y sus valores de diversidad.

\begin{tabular}{|c|c|c|c|c|c|c|c|}
\hline Petén & $\begin{array}{l}\text { Distancia } \\
\text { del mar }\end{array}$ & Diámetro & Area & $\mathrm{N}_{0}$ & $\mathrm{~N}_{1}$ & $\mathrm{~N}_{2}$ & E \\
\hline Petén 1 & $4969 \mathrm{~m}$ & $280 \mathrm{~m}$ & 6.16 ha & 11 & 2.88 & 2.17 & 0.44 \\
\hline Petén 2 & $4640 \mathrm{~m}$ & $320 \mathrm{~m}$ & 8.04 ha & 8 & 3.28 & 2.52 & 0.57 \\
\hline Petén 3 & $11440 \mathrm{~m}$ & $400 \mathrm{~m}$ & $12.56 \mathrm{ha}$ & 12 & 4.27 & 2.92 & 0.58 \\
\hline Petén 4 & $8800 \mathrm{~m}$ & $560 \mathrm{~m}$ & 24.63 ha & 12 & 6.88 & 5.39 & 0.77 \\
\hline
\end{tabular}

$\begin{array}{ll}N_{0}=\text { Riqueza florística. } & N_{2}=1 / H \text { (Inverso del índice de Simpson) } \\ N_{1}=\text { Antilog } H^{\prime} \text { (según Shannon-Wiener) } & E^{2}=\text { Indice de Equidad ( } H^{\prime} / H \text { max.) }\end{array}$

esta diferencia. Se puede apreciar que los índices de Shannon-Wiener y de Simpson manifiestan una marcha muy similar y varían de forma esencialmente paralela, en tanto que la riqueza florística $\left(\mathrm{N}_{0}\right)$ lo hace de manera muy diferente.

Es necesario hacer notar que la diferencia entre los valores de diversidad de los petenes 2 y 3 se debe en esencia al incremento en el número de especies, pues el monto de equidad en ambos es muy similar. Cabe advertir que este cambio se manifiesta más fuertemente bajo el índice de Shannon-Wiener. Por el contrario, la diferencia entre los valores de diversidad de los petenes 3 y 4 se debe exclusivamente a la distribución de abundancias, ya que el número de especies permanece constante. Los números obtenidos con el empleo de ambos índices se modifican de forma similar, pero en este caso el cambio se presenta más acentuado bajo el de Simpson.

Por último, se aprecia que al aumentar el tamaño de los petenes se incrementa la diversidad de sus árboles. Estableciendo una regresión entre el área y la diversidad, se obtienen valores significativos en el coeficiente correspondiente.

El cuadro 2 presenta los valores de importancia de las especies de cada petén, de acuerdo con el índice combinado propuesto por Curtis (1959). Se detallan además los valores de densidad, frecuencia y área basal relativos que están involucrados.

Si se determinara el valor de importancia de las especies considerando por separado cada uno de estos parámetros, el orden jerárquico encontrado diferiría notablemente en función del parámetro que se utilice. Es decir, que el orden resultante al considerar únicamente la densidad sería muy distinto al que se obtiene si se emplea el área basal y ambos diferirían del encontrado al utilizar el índice combinado.

La figura 2 muestra las curvas de dominancia-diversidad que resultan al considerar como estimadores de la abundancia $\left(p_{\mathrm{i}}\right)$ a los distintos parámetros antes mencionados, tomando como ejemplo para ello al petén 1. Se observa que, además de los cambios en el orden jerárquico, las curvas de dominancia que se obtienen bajo el uso de los distintos parámetros difieren notablemente.

En función de estos problemas, se decidió utilizar el índice de Curtis para generar las curvas de dominancia-diversidad de las cuatro comunidades (Fig. 3). Cada una de éstas se comparó con una curva teórica según el modelo de Motomura (citado en Pielou, 1975) 


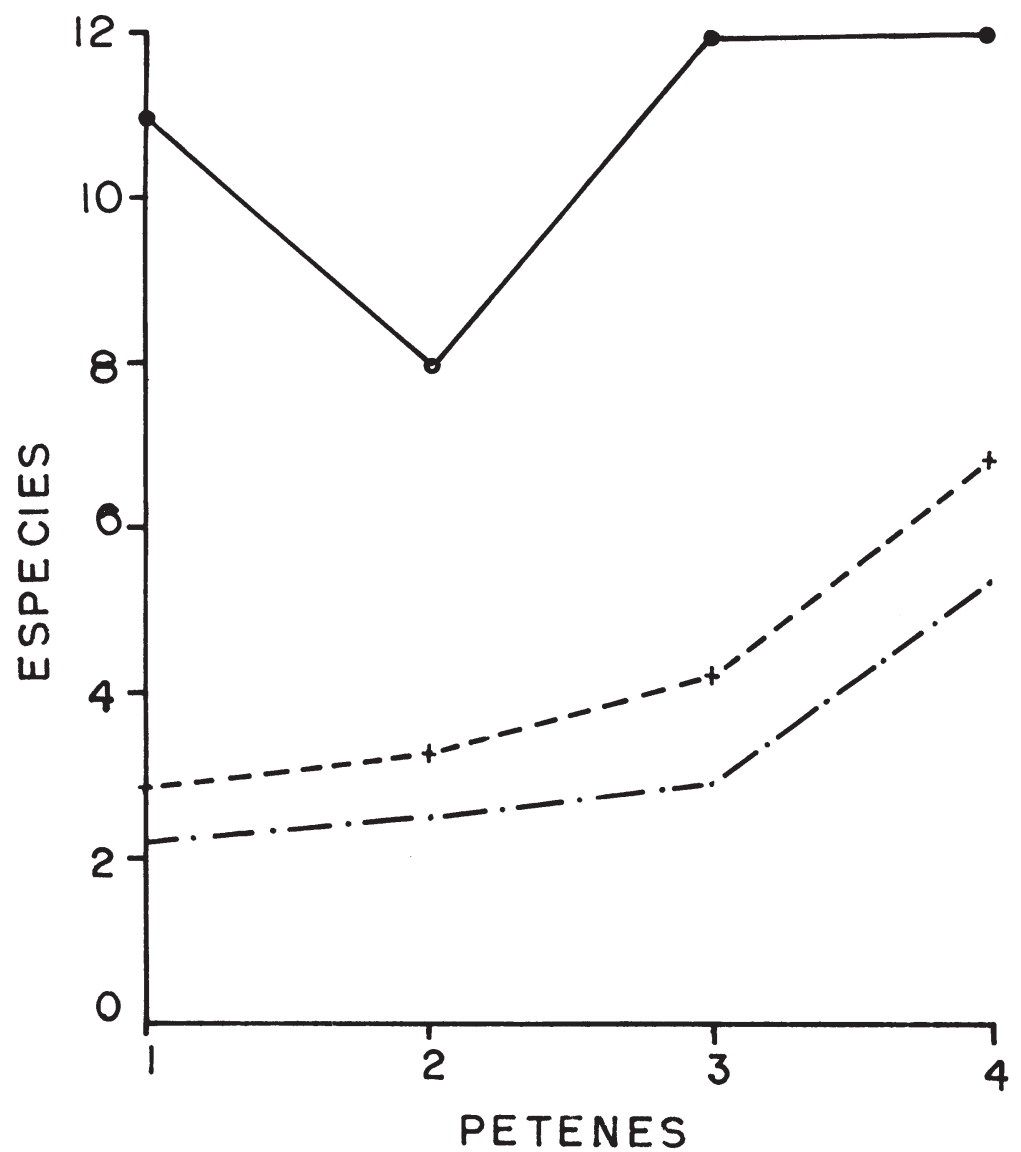

Fig. 1. Comportamiento de los índices de diversidad en los petenes. Riqueza florística (-); $N_{1}$ ShannonWiener (---); $\mathrm{N}_{2}$ Simpson (--.).

y se utilizó una prueba de "F" para estimar la bondad del ajuste. Los valores resultantes del análisis de varianza fueron altamente significativos (Cuadro 3).

De la misma manera, las curvas de dominancia-diversidad se compararon con un modelo de tipo lineal, mediante un análisis de regresión. En todos los casos el ajuste obtenido con el modelo de Motomura fue más satisfactorio, como muestran los valores de "F" que resultan del análisis. No obstante, se obtuvieron diferencias significativas sólo para los petenes 1,3 y 4 (Cuadro 3 ).

Las curvas obtenidas para todas las comunidades se compararon mediante una prueba de Kolmogorov-Smirnov y los resultados se muestran en el Cuadro 4. Se observa que ninguna de las comunidades difiere significativamente de las otras en su patrón de distribución de abundancias. 
Acta Botánica Mexicana (1995), 31:73-84

Cuadro 2. Valores de importancia de las especies en los 4 petenes.

\begin{tabular}{|c|c|c|c|c|}
\hline Especies & $\begin{array}{l}\text { Densidad } \\
\text { relativa }\end{array}$ & $\begin{array}{l}\text { Frecuencia } \\
\text { relativa }\end{array}$ & $\begin{array}{l}\text { Area } B . \\
\text { relativa }\end{array}$ & $\begin{array}{l}\text { Valor de } \\
\text { importancia }\end{array}$ \\
\hline \multicolumn{5}{|l|}{ Petén 1} \\
\hline Sabal yара & 26.84 & 22.85 & 35.70 & 85.39 \\
\hline Bravaisia tubiflora & 62.13 & 20.01 & 1.40 & 83.53 \\
\hline Manilkara zapota & 5.88 & 20.01 & 29.30 & 55.18 \\
\hline Swietenia macrophylla & 1.40 & 11.40 & 11.26 & 24.06 \\
\hline Laguncularia racemosa & 0.35 & 2.80 & 13.50 & 16.65 \\
\hline Ficus maxima & 0.70 & 5.70 & 8.13 & 14.53 \\
\hline Pisonia aculeata & 0.70 & 5.70 & 0.17 & 6.57 \\
\hline Neea psychotrioides & 0.70 & 2.80 & 0.01 & 3.51 \\
\hline Annona glabra & 0.35 & 2.80 & 0.29 & 3.44 \\
\hline Talisia oliviformis & 0.35 & 2.80 & 0.18 & 3.33 \\
\hline Metopium brownei & 0.35 & 2.80 & 0.01 & 3.16 \\
\hline \multicolumn{5}{|l|}{ Petén 2} \\
\hline Sabal yapa & 32.74 & 20.01 & 37.30 & 90.04 \\
\hline Laguncularia racemosa & 4.27 & 20.01 & 54.20 & 78.57 \\
\hline Bravaisia tubiflora & 53.38 & 20.01 & 1.83 & 75.21 \\
\hline Ficus maxima & 4.27 & 15.01 & 1.15 & 20.42 \\
\hline Annona glabra & 2.84 & 12.50 & 0.39 & 15.73 \\
\hline Manilkara zapota & 1.06 & 5.01 & 1.55 & 7.62 \\
\hline Ficus tecolutensis & 0.70 & 2.50 & 3.38 & 6.58 \\
\hline Neea psychotrioides & 0.70 & 5.01 & 0.05 & 5.76 \\
\hline \multicolumn{5}{|l|}{ Petén 3} \\
\hline Bravaisia tubiflora & 52.42 & 19.00 & 1.06 & 72.48 \\
\hline Sabal yapa & 22.01 & 19.00 & 18.53 & 59.54 \\
\hline Manilkara zapota & 12.77 & 16.60 & 26.64 & 56.01 \\
\hline Ficus maxima & 1.76 & 4.76 & 25.09 & 31.61 \\
\hline Swietenia macrophylla & 1.32 & 4.76 & 25.18 & 31.26 \\
\hline Laguncularia racemosa & 3.08 & 7.10 & 2.63 & 12.81 \\
\hline Tabebuia rosea & 1.76 & 9.52 & 0.06 & 11.34 \\
\hline Talisia oliviformis & 2.20 & 7.10 & 0.63 & 9.93 \\
\hline Annona glabra & 1.32 & 4.76 & 0.08 & 6.16 \\
\hline Bursera simaruba & 0.44 & 2.38 & 0.05 & 2.87 \\
\hline Ardisia escallonioides & 0.44 & 2.38 & 0.02 & 2.84 \\
\hline Diospyros digyna & 0.44 & 2.38 & 0.02 & 2.84 \\
\hline
\end{tabular}


Cuadro 2. Continuación.

\begin{tabular}{|lcccc|}
\hline Especies & $\begin{array}{c}\text { Densidad } \\
\text { relativa }\end{array}$ & $\begin{array}{c}\text { Frecuencia } \\
\text { relativa }\end{array}$ & $\begin{array}{c}\text { Area B. } \\
\text { relativa }\end{array}$ & $\begin{array}{c}\text { Valor de } \\
\text { importancia }\end{array}$ \\
\hline Petén 4 & & & & \\
Rhizophora mangle & 24.32 & 21.60 & 30.93 & 76.85 \\
Sabal yapa & 18.92 & 18.90 & 17.76 & 55.58 \\
Manilkara zapota & 10.80 & 13.50 & 17.92 & 42.22 \\
Bravaisia tubiflora & 27.02 & 10.80 & 0.44 & 38.26 \\
Laguncularia racemosa & 3.60 & 5.40 & 23.75 & 32.75 \\
Ficus tecolutensis & 5.40 & 5.40 & 4.08 & 14.88 \\
Metopium brownei & 2.70 & 5.40 & 3.82 & 11.98 \\
Swietenia macrophylla & 1.80 & 5.40 & 0.12 & 7.32 \\
Elaeodendron xylocarpum & 1.80 & 5.40 & 0.05 & 7.25 \\
Talisia oliviformis & 1.80 & 2.70 & 0.95 & 5.45 \\
Tabebuia rosea & 0.90 & 2.70 & 0.16 & 3.76 \\
Dalbergia glabra & 0.90 & 2.70 & 0.01 & 3.61 \\
\hline
\end{tabular}

\section{DISCUSION}

Los resultados obtenidos mediante el uso de los diferentes índices de diversidad corroboran lo expresado por Hill (1973), y por Peet (1974) en relación a la sensibilidad de cada uno de ellos a los cambios en el número de especies y en la distribución de sus abundancias.

Es claro que la riqueza florística se comporta de manera muy diferente a los otros índices y en definitiva, no puede ser considerada como un medida de diversidad, si entendemos por diversidad, a la heterogeneidad de la comunidad. Es evidente que la riqueza florística depende en gran proporción del tamaño de muestra utilizado (Krebs, 1985).

Los valores obtenidos empleando los índices de Simpson y de Shannon-Wiener (Cuadro 1 y Fig. 1) muestran un comportamiento muy similar a pesar de sus diferencias en sensibilidad. Ya Peet (1974) había mostrado tal paralelismo al usar estos coeficientes en comunidades de aves e indicó que el fenómeno se presenta cuando las comunidades no difieren grandemente en sus patrones de dominancia.

Es importante destacar que los índices, al ser transformados de acuerdo con Hill (1973), dan valores de número de especies aparentes bastante bajos, lo cual sugiere una dominancia muy marcada. Por ejemplo, el petén 1 presenta únicamente 2 ó 3 especies aparentes, a pesar de que existen 11 en la comunidad. Por su parte, el petén 4, con un mayor valor en el índice de equidad, registra 5 a 7 especies aparentes, lo que equivale a la mitad de las que allí se encuentran. Lo anterior muestra, además, que la dominancia disminuye y la diversidad se incrementa conforme aumenta el tamaño del petén.

Es necesario señalar que la preferencia del uso de uno u otro índice depende en gran medida de los objetivos que se persigan en el trabajo. El de Simpson valora fuertemente la distribución de abundancias y esto se puede expresar como el grado de 


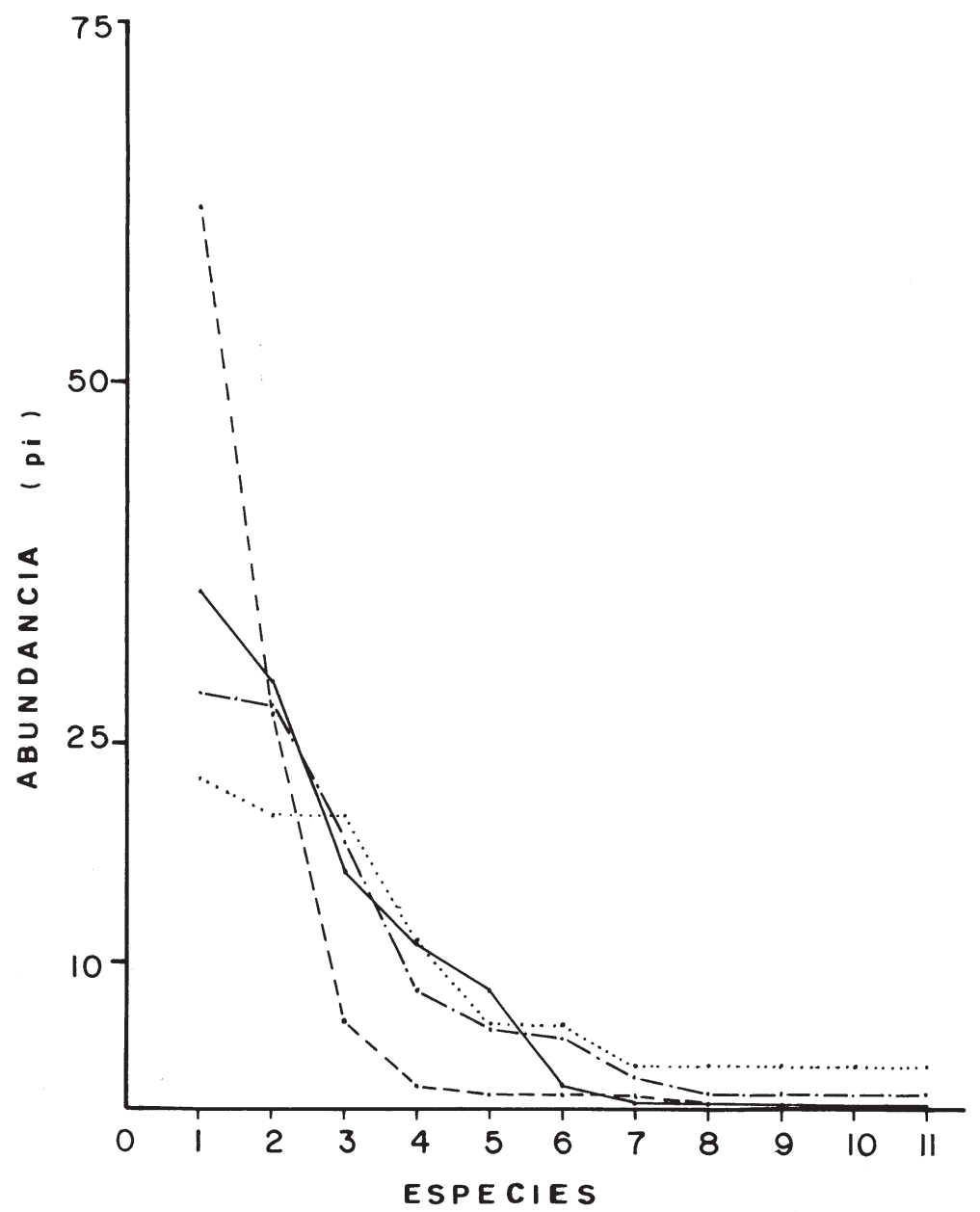

Fig. 2. Curvas de dominancia obtenidas al emplear como estimador de la abundancia a 4 distintas variables. Densidad relativa (---); Area basal rel. (-); Frecuencia rel. ( ....); Valor de importancia rel. $(\mathrm{VIR} / 3)(\cdot-)$.

dominancia, en tanto que el de Shannon-Wiener da mayor peso a la riqueza florística, con lo cual la presencia de "plantas raras" puede ser importante.

En comunidades de selva muchas especies de árboles suelen tener una distribución muy dispersa, por lo que son frecuentes a nivel de cuadros de muestreo, pero poco abundantes en cada uno, por lo cual se registran como especies raras. Estas no pueden ser consideradas como ocasionales, ya que a pesar de que el tamaño de sus poblaciones 
Durán: Diversidad Florística de los Petenes de Campeche
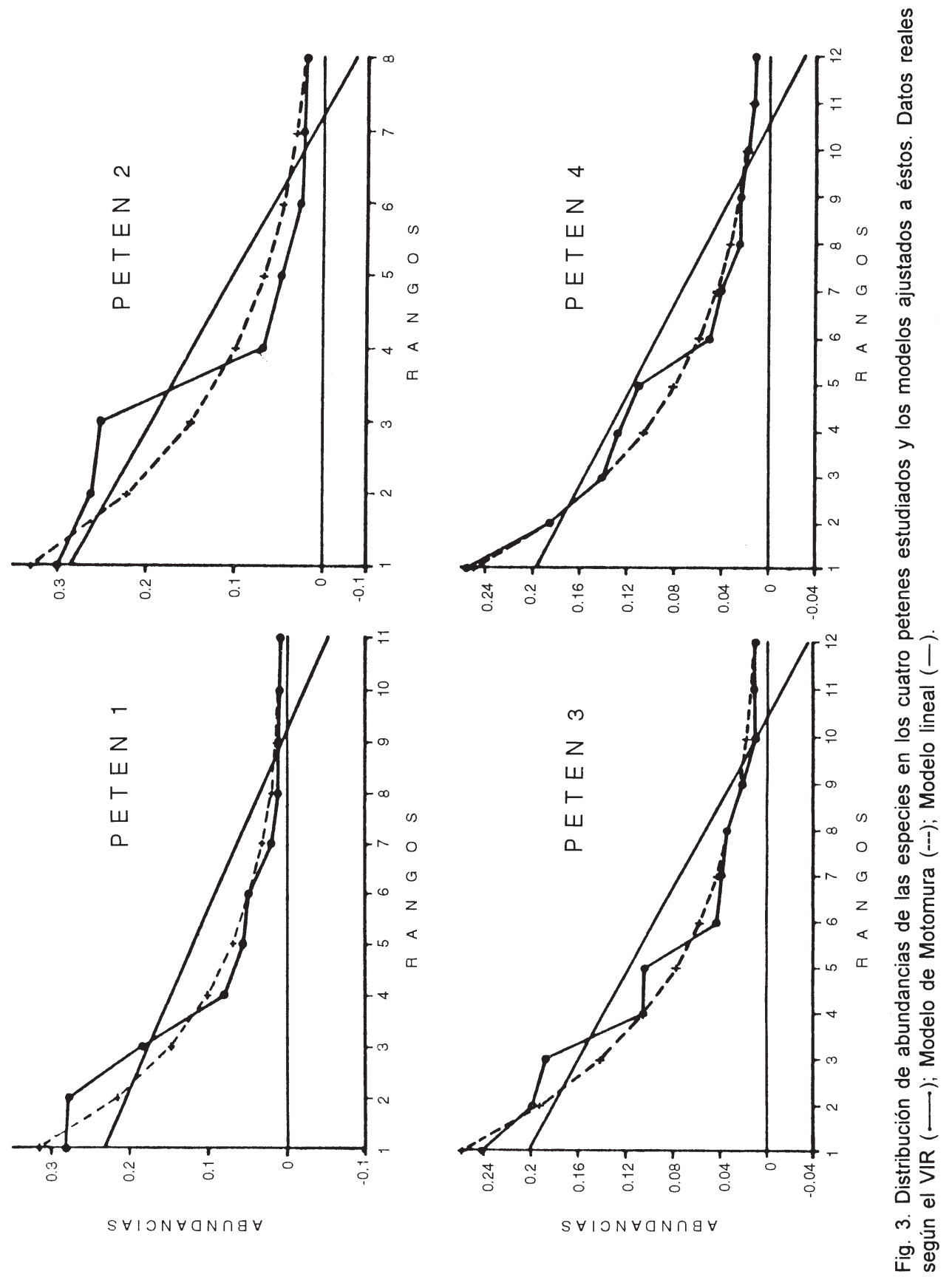
Cuadro 3. Comparación entre los modelos empleados.

\begin{tabular}{|l|rrrr|}
\hline Petén & $\begin{array}{l}\text { Modelo de } \\
\text { Motomura }\end{array}$ & $\begin{array}{l}\text { Modelo } \\
\text { lineal }\end{array}$ & $\begin{array}{l}\text { Cociente } \\
\mathrm{F}(1) / \mathrm{F}(\mathrm{m})\end{array}$ & \\
\hline & & & & \\
Petén 1 & 137.72 & 31.076 & 3.6612 & $*$ \\
Petén 2 & 36.89 & 30.504 & 1.1751 & N.S. \\
Petén 3 & 218.42 & 62.019 & 3.1716 & $*$ \\
Petén 4 & 421.20 & 59.851 & 6.1731 & $*$ \\
\hline
\end{tabular}

sea pequeño, en realidad son especies que siempre están presentes y posiblemente jueguen un papel importante.

En definitiva, el uso del método de Hill (1973) hace que los índices mencionados sean más comprensibles, ya que se interpreta la diversidad en términos del número de especies que aparentemente están participando en la estructuración de la comunidad. Las expresiones logarítmicas que resultan de las fórmulas de Simpson y de Shannon-Wiener no son fáciles de interpretar.

En cuanto a los valores de importancia, parece claro que los resultados dependerán fuertemente del parámetro utilizado por cada autor y para ello debe considerarse el tipo de comunidad que se pretenda estudiar. Al parecer, la cobertura ha sido empleada con mucho éxito en los estudios de vegetación realizados en desiertos, sin embargo en las selvas no parece ser tan adecuada. En las comunidades en cuestión se presentan especies vegetales muy distintas en formas de crecimiento y tamaño, por lo cual la elección de uno u otro parámetro tiene que ser muy cuidadosa.

Es evidente que si comparamos una especie arbórea del dosel superior de la selva con una del sotobosque, mediante el parámetro densidad, encontraremos probablemente muchos individuos de la planta pequeña y muy pocos de la que alcanza el dosel, obteniendo que en la curva de distribución de abundancias la especie del sotobosque ocuparía un rango mayor que la del dosel. Resultados muy distintos se lograrían si comparáramos a estas especies con base en la cobertura, la biomasa o el área basal.

Sin duda, es difícil la elección objetiva del mejor parámetro como indicador de la dominancia, pero se puede aminorar el problema si se seleccionan especies de tamaños comparables, o bien, utilizando un índice que involucre tanto aspectos de biomasa y número de individuos, como la constancia con que se presenta cada componente de la comunidad.

En cuanto a la distribución de abundancias, las curvas que mejor se ajustan a los datos de campo corresponden al modelo de Motomura, el cual predice que la repartición del nicho dependerá en gran medida de la forma como se haya dado la colonización y que todas las especies tienen igual capacidad de apropiación de los recursos. Estas curvas se han ajustado con éxito a comunidades que se encuentran en procesos sucesionales (Bazzaz, 1975). Por su parte Whittaker (1972) encontró en bosques de pino, que sólo en las etapas más tempranas se presenta una dominancia suficientemente grande para que la distribución de abundancias se ajuste adecuadamente al modelo de Motomura. 
Cuadro 4. Pruebas de Kolmogorov-Smirnov.

\begin{tabular}{|l|cccc|}
\hline & Petén 1 & Petén 2 & Petén 3 & Petén 4 \\
\hline Petén 1 & ---- & & & \\
Petén 2 & 0.06573 & ---- & & \\
Petén 3 & 0.12303 & 0.186 & --- & \\
Petén 4 & 0.16483 & 0.23056 & 0.04456 & --- \\
\hline
\end{tabular}

Un modelo alternativo, con el cual frecuentemente se compara el anterior, es el de MacArthur, también denominado "de la vara partida" (citado en Pielou, 1975). Este predice una distribución más equitativa de las abundancias de las especies y una división aleatoria del nicho, como producto de su desigual capacidad para aprovechar los recursos existentes. Tal modelo parece ajustarse mejor a las comunidades donde se presume que la competencia es el factor que determina su estructura. Para los petenes estudiados, el modelo de MacArthur fue poco satisfactorio.

Los resultados parecen apoyar la idea de que los petenes son comunidades que se encuentran en distintas etapas sucesionales (Barrera, 1982; Rico-Gray, 1982), aun cuando este tipo de sucesión esté determinado por la dinámica de emersión de la Península de Yucatán y por los consecuentes cambios en la humedad y la salinidad de cada isla. Así, parece ser que los petenes más distantes del mar tienden a ser más diversos y a presentar una dominancia menos marcada.

El uso de los modelos de diversidad-abundancia resulta ser un instrumento descriptivo, que brinda una imagen de cómo se da la repartición de los recursos en la comunidad, sin importar la identidad de las especies. Sin embargo, Pielou (1975) señala que el hecho de que un conjunto de datos se ajuste a determinado modelo, puede indicar tan sólo la gran variedad de formas que puede tomar una familia de curvas. Por lo tanto, sugiere como una mejor aproximación al entendimiento de los patrones de dominancia, comparar las curvas de distribución de abundancias de las distintas comunidades.

Mediante la prueba de Kolmogorov-Smirnov se encontró que en ningún caso se registraron diferencias significativas, es decir, que todos los petenes presentan un patrón de dominancia semejante, lo que concuerda con lo expresado por Peet (1974) acerca del comportamiento paralelo de los índices de diversidad en comunidades que manifiestan patrones de dominancia similares.

Por último, es importante señalar que aun cuando el uso de estas herramientas estadísticas nos permite comparar las comunidades en lo relativo a la abundancia de las especies y la forma como se distribuyen los recursos, es necesario tener cuidado en la elección de los modelos a utilizar, de los parámetros que se pretenden estudiar y de las conclusiones que se pueden derivar.

Los modelos de Motomura y MacArthur sólo son simplificaciones de un fenómeno multivariado de apropiación de los recursos por las distintas especies, por lo que deben considerarse únicamente como herramientas exploratorias. 


\section{AGRADECIMIENTOS}

Deseo manifestar mi más sincero agradecimiento al Dr. Exequiel Ezcurra y a los Bióls. Oscar Briones y Hugo Riemman, por los valiosos consejos y las fuertes críticas que me hicieron durante la preparación del manuscrito.

\section{LITERATURA CITADA}

Barrera, A. 1982. Los petenes del noroeste de Campeche. Su exploración ecológica en perspectiva. Biótica 7(2): 163-169.

Bazzaz, F. A. 1975. Plant species diversity in oldfield successional ecosystems in southern Illinois. Ecology 56: 485-488.

Curtis, J. T. 1959. The vegetation of Wisconsin. An ordination of plant communities. Univ. of Wisconsin Press. Madison. $657 \mathrm{pp}$.

Durán, R. 1987. Descripción y análisis de la estructura y composición de la vegetación de los petenes del noroeste de Campeche. Biótica 12(3): 181-192.

Hill, M. O. 1973. Diversity and evenness: a unifying notation and its consequences. Ecology 54: 427432.

Krebs, C. J. 1985. Ecología. Estudio de la distribución y la abundancia. 2a. ed. Harper \& Row Latinoamericana. México, D.F. pp. 495-536.

Olmsted, I., A. López-Ornat y R. Durán. 1983. Vegetación de Sian Ka’an: Estudios preliminares de una zona en Quintana Roo, propuesta como Reserva de la Biósfera. Centro de Investigaciones de Quintana Roo, Secretaría de Desarrollo Urbano y Ecología. Cancún, Quintana Roo. pp. 6384.

Peet, R. K. 1974. The measurement of species diversity. Ann. Rev. Ecol. Syst. 5: 285-307.

Pielou, E. C. 1975. Ecological diversity. Wiley. Nueva York. 165 pp.

Rico-Gray, V. 1982. Estudio de la vegetación de la zona costera inundable del noroeste del estado de Campeche, México. Los Petenes. Biótica 7(2): 171-190.

Whittaker, R. H. 1972. Evolution and measurement of species diversity. Taxon 21: 213-251. 